\title{
Composition en acide alphalinolénique des esters de cholestérol plasmatiques : un marqueur de consommation chez l'homme
}

\section{Assessing the level of alphalinolenic acid in plasma cholesteryl esters in humans}

Oléagineux, Corps Gras, Lipides. Volume 9, Numéro 4, 245-8, Juillet - Août 2002, Dossier : Equilibre entre acides gras insaturés : contributions à l'étude de la prévention des maladies cardio-vasculaires

Auteur(s) : Nicole COMBE, Bernadette DELPLANQUE, Stéphanie TANGUY, Carole BOUE-VAYSSE, François MENDY, Brigitte LE ROY, Evelyne FENART, Département de biochimie et de nutrition, ITERG, Université Bordeaux I, 33405 Talence Cedex, France.

Author(s) : Nicole COMBE, Bernadette DELPLANQUE, Stéphanie TANGUY, Carole BOUE-VAYSSE, François MENDY, Brigitte LE ROY, Evelyne FENART

Résumé : Le lien existant entre qualité et quantité des acides gras de l'alimentation, et santé est de plus en plus évident et défini, comme l'indique la revue des travaux plus spécifiquement consacrés aux implications dans le risque de maladies cardiovasculaires (MCV) (cf. premier exposé de ce dossier). Néanmoins, si un grand nombre d'études épidémiologiques ou d'intervention ont permis d'aboutir à un consensus qui s'est traduit, en France, par la publication en 2001 des Apports Nutritionnels Conseillés [1], un débat perdure au sein de la communauté scientifique internationale sur les meilleurs apports en acides gras monoinsaturés versus poly-insaturés [2, 3]. Les études récentes de Rudel et al. [4-6] indiquent en effet que des singes nourris avec un régime très enrichi en acide oléique (18:1n-9 : $24 \%$ de l'apport énergétique) développent une athérosclérose des artères coronaires qui pourrait être en relation avec l'accumulation subséquente d'oléate de cholestérol dans les LDL aux dépens du linoléate de cholestérol. Par ailleurs, eu égard aux effets bénéfiques reconnus à l'acide alphalinolénique (18:3n-3) vis-à-vis des risques d'infarctus du myocarde, de cardiopathie ischémique [7-9] et d'accident vasculaire cérébral $[10,11]$, le niveau de 18:3n-3 dans les esters de cholestérol (EC) du plasma apparaît comme un bon indicateur de protection. En effet, l'étude conduite par Sandker et al. [12], sur deux groupes d'hommes âgés, issus des cohortes crétoises (Seven Countries Study) et hollandaises (Zutphen Study), a permis de faire émerger l'intérêt de ce marqueur biologique. En moyenne, le pourcentage de 18:3n-3 mesuré dans les EC plasmatiques des Crétois était significativement plus élevé comparé à celui des Hollandais $(0,9 \%$ vs $0,32 \%$ des acides gras), dont la cohorte présentait un taux de mortalité coronarienne 5 fois plus grand que celui de la cohorte crétoise. En outre, l'étude cas-témoins de Simon et al. [10] a montré en 1995 qu'un taux plasmatique de 18:3n-3 élevé était associé à une réduction de $37 \%$ du risque d'accident vasculaire cérébral. Après analyse multivariée pour prendre en compte les facteurs de confusion, susceptibles d'interférer dans le risque étudié, seul le pourcentage de 18:3n-3 dans les EC plasmatiques restait inversement associé au risque d'accident vasculaire cérébral $(p<0,05)$. De même, l'étude d'intervention de Bemelmans et al. [13], destinée à la prévention MCV chez des sujets à risques, a récemment montré que le pourcentage de 18:3n-3 dans les EC du plasma était inversement associé à la pression diastolique $(p<0,05)$. II ressort de l'ensemble de ces données que le niveau d'acide alphalinolénique souhaitable dans les EC du plasma se situerait entre 0,4 et 0,9\% par rapport aux acides gras totaux liés au cholestérol. 
Summary : The level of alpha-linolenic acid (ALA) in plasma cholesteryl esters (CE) could be a reliable biomarker to assess the risks of coronary heart disease and stroke. Epidemiologic studies have shown that these diseases occurred in subjects whose ALA content in CE ( $0.3 \%$ of total fatty acids) was lower than that in healthy subjects (0.4-0.9\%). The aim was to study the effect of the intake of oleic acid (OA), ranging from 11 to $16 \%$ of total dietary energy. Previous studies have shown that diets rich in monounsaturated fat promoted atherosclerosis when compared to diets substituted with isocaloric amounts of saturated or polyunsaturated fat, and cholesteryl oleate replaced cholesteryl linoleate as the major cholesterol ester. In the present study, the linoleic acid/linolenic acid ratio (LA/ALA) of the diets was maintained between 5.0 and 7.9. Thirty healthy men (monks) received 3 controlled diets for 4 months one after the other separated by a washout period. Different proportions of ALA, LA and $O A$ in dietary fat were obtained from different combinations of oils (high oleic sunflower/sunflower/rapeseed). Lipids were 30, 34 and 35\% energy in diets I, II and III, respectively. Diet I provided $28.2 \mathrm{~g} / \mathrm{d}$ OA (=11\% en), $9.0 \mathrm{~g} / \mathrm{d}$ LA and $1.4 \mathrm{~g} / \mathrm{d}$ ALA; diet II: $36.5 \mathrm{~g} / \mathrm{d}$ OA (=13\% en), $13.0 \mathrm{~g} / \mathrm{d}$ LA and $2.6 \mathrm{~g} / \mathrm{d}$ ALA; diet III: $43.3 \mathrm{~g} / \mathrm{d}$ OA (=16\% en), $11.8 \mathrm{~g} / \mathrm{d}$ LA and $1.5 \mathrm{~g} / \mathrm{d}$ ALA. The OA/LA ratio was 3.1 (diet I), 2.8 (diet II) and 3.6 (diet III). The LA/ALA ratio was 6.4 (diet I), 5.0 (diet II) and 7.9 (diet III). After each diet, fatty acid compositions of plasma cholesteryl esters were the following: i) OA: $19.42 \pm 1.50 \%$ (diet I); $16.39 \pm 1.30 \%$ (diet II); $19.47 \pm 1.45 \%$ (diet III); ii) LA: $51.67 \pm 2.85 \%$ (diet I); $55.12 \pm 3.89 \%$ (diet II); $52.96 \pm 3.10 \%$ (diet III); iii) ALA: $0.70 \pm 0.12 \%$ (diet I); $0.68 \pm 0.12 \%$ (diet II); $0.63 \pm 0.10 \%$ (diet III). These results show that these different supplies of OA, LA and ALA allow to obtain a favorable value of ALA $(>0.6 \%)$ in plasma CE. Compared with a standard French population, $18: 3 n-3$ increased by $57 \%$ and $20: 5 n-3$ doubled ( $1.2 \%$ vs $0.6 \%$ of total fatty acids) in plasma cholesterol esters of subjects fed these diets.

Keywords : alphalinolenic acid, cholesterol ester, plasma, diet.

\section{ARTICLE}

De façon générale, l'acide 18:3n-3 s'accumule peu dans les tissus contrairement à l'acide linoléique (18:2n-6). On sait que la biodisponibilité cellulaire de l'acide 18:3n-3 est très fortement limitée par sa grande capacité à être rapidement betaoxydé, 2 à 16 fois plus vite en 6 heures que d'autres acides gras poly-insaturés tels que les acides linoléique, gammalinolénique et arachidonique [14]. Ainsi, chez le rat, 64 \% de la quantité de 18:3n-3 administrée sous forme libre peuvent être « brûlés » en 24 heures. Bien que ces chiffres soient atteints en 7 jours chez l'homme, on considère actuellement [15, 16] que moins de $5 \%$ du 18:3n-3 consommé sont métabolisés en 22:6n-3 (acide docosahexaénoïque : DHA). Ce " gaspillage » de précurseur s'explique en partie par l'efficacité des mécanismes enzymatiques impliqués dans la beta oxydation mitochondriale vis-à-vis de l'acide 18:3n-3 [17]. Bien entendu, les concentrations en acide linoléique et acides gras poly-insaturés à très longues chaînes du régime influencent également la biodisponibilité de l'acide alphalinolénique, en raison des voies métaboliques communes aux deux séries d'acides gras poly-insaturés n- 6 et n-3.

Dans ce contexte, quelques études d'intervention témoignent de conditions nutritionnelles qui permettent d'accroître le niveau d'acide alphalinolénique dans les esters de cholestérol. Parmi cellesci, l'étude de Mantzioris et al. [18] visait à déterminer le potentiel de l'huile de graines de lin pour 
augmenter les concentrations tissulaires en 20:5n-3 (acide eicosapentaénoïque : EPA). Deux groupes de sujets sains ont été comparés après 4 semaines de régime. Le groupe "contrôle " a conservé ses habitudes alimentaires (type occidental), caractérisées par des apports en 18:2n-6 et 18:3n-3 représentant respectivement 7,8 et $0,4 \%$ de l'apport énergétique total (AET). Pour le groupe expérimental, l'acide 18:2n-6 était abaissé à 3,3 \% AET et l'acide 18:3n-3 relevé à 5,3\% AET. Au terme de la période d'intervention, le pourcentage de 18:3n-3 dans les EC plasmatiques du groupe expérimental était multiplié environ par 7 par rapport au groupe contrôle $(3,0 \%$ vs $0,4 \%$ des acides gras). De même, les proportions d'EPA étaient significativement $(p<0,05)$ augmentées $(2,0 \%$ vs 0,8 $\%)$, en revanche, celles de DHA (22:6n-3) n'étaient pas modifiées ( $0,6 \%$ vs $0,5 \%)$. Pour obtenir ces résultats, il a fallu faire passer le rapport 18:2n-6/18:3n-3 de 19,5 (groupe contrôle) à 0,62 (groupe expérimental), en substituant les corps gras usuels de l'alimentation par de l'huile de lin désodorisée (55 \% de 18:3n-3 et 18,3\% de 18:2n-6) et une margarine à base d'huile de lin ( $22,6 \%$ de $18: 3 n-3$ et $8,3 \%$ de 18:2n-6). L'étude de Valsta et al. [19], conduite chez 40 sujets sains, a montré la capacité de I'huile de colza à compenser la perte en EPA du plasma induite par la restriction alimentaire de poissons. Le régime " colza " testé apportait 2,2 \% AET de 18:3n-3 et 6,6 \% de 18:2n-6 (soit un rapport 18:2n-6/18:3n-3 $=3$ ). Il permettait d'augmenter de $30 \%$ les proportions de $18: 3 n-3$ et de maintenir celles d'EPA dans les EC du plasma. Les résultats de la Margarin Study, inspirée de la Lyon Diet Heart Study, ont été publiés récemment [20]. Cette étude d'intervention concernait 124 hommes et 158 femmes qui présentaient des facteurs de risque MCV. Les sujets qui ont suivi pendant un an le régime enrichi en 18:3n-3 (2,1 \% vs 0,5\% AET avant l'intervention) présentaient une augmentation significative $(p<0,01)$ des pourcentages de 18:3n-3 et d'EPA dans les EC plasmatiques ; ceux-ci passaient respectivement de 0,52 à $0,99 \%$ pour l'acide 18:3n-3 et de 1,02 à $1,37 \%$ pour l'EPA.

Enfin si, au plan pratique, on s'interroge sur la validité de la composition en 18:3n-3 dans les EC plasmatiques comme indicateur biologique de l'apport en 18:3n-3, l'exploitation des résultats de I'ARIC Study, fondée sur les données de 3570 sujets participant à cette étude prospective, a montré l'existence d'une corrélation entre le pourcentage de 18:3n-3 dans les EC du plasma et l'apport en 18:3n-3, exprimé en pourcentage des lipides totaux consommés $(r=0,30$; $p<0,01)$ [21]. Dans une étude d'intervention chez des enfants prématurés qui recevaient une formule enrichie en 18:3n-3, Babin et al. [22] ont trouvé une corrélation très significative $(r=0,66 ; p$ $<0,0001$ ) entre le pourcentage de 18:3n-3 présent dans les EC du plasma au $15^{\mathrm{e}}$ jour de l'étude et la quantité cumulée de 18:3n-3 ingérée pendant cette période.

Dans le cadre de cette étude dont l'objectif était d'évaluer, chez l'homme sain, les effets nutritionnels de la modulation du rapport : acide oléique/acides gras poly-insaturés, en relation avec les paramètres du risque $\mathrm{MCV}$, nous nous sommes attachés à suivre l'évolution de la composition en acide alphalinolénique, et plus généralement celle des acides gras poly-insaturés, dans les EC plasmatiques, pour leur implication forte dans les fonctions plasmatiques et cellulaires.

\section{Sujets et méthodes}

\section{Sujets et protocole nutritionnel}

Les caractéristiques des sujets participant à cette étude ainsi que les détails du protocole nutritionnel (composition en huiles et acides gras des régimes, événements) sont fournis dans le premier exposé de ce dossier). Nous en rappelons brièvement quelques éléments. Une quarantaine de sujets 
masculins ont consommé pendant 4 mois un régime (dit OL-13\%) à $34 \%$ AET de lipides, dont $13 \%$ de $18: 1 n-9,4,5 \%$ de $18: 2 n-6$ et $0,91 \%$ de $18: 3 n-3$. À l'issue de cette période, deux groupes ont été constitués pour deux interventions croisées (régimes OL-11\% et OL-16\%) de 4 mois chacune. Les caractéristiques de ces régimes sont les suivantes : $30 \%$ AET de lipides, dont $11 \%$ de 18:1n-9, 3,6 \% de $18: 2 n-6$ et $0,55 \%$ de $18: 3 n-3$ pour le régime OL-11 \% et $35 \%$ AET de lipides, dont $16 \%$ de $18: 1 n$ $9,4,4 \%$ de 18:2n- 6 et $0,55 \%$ de 18:3n-3 pour le régime OL-16\%. Le rapport 18:2n-6/18:3n-3 dans ces trois régimes variait peu (compris entre 5 et 8). L'acide alphalinolénique était apporté par l'huile de colza (répartition du 18:3n-3 au sein des TG : $58 \%$ en position sn 2 et $42 \%$ en positions $s n 1+s n$ 3). Au terme de chaque période, un échantillon de sang des sujets à jeun était prélevé et centrifugé immédiatement. Le plasma était conservé, congelé à $30^{\circ} \mathrm{C}$, jusqu'à analyse.

\section{Analyse des acides gras des esters de cholestérol plasmatiques}

Les techniques d'analyse ont été décrites par ailleurs [23]. Brièvement, après extraction des lipides totaux selon la technique de Folch et al., la fraction des EC a été isolée par chromatographie sur couche mince et ses acides gras analysés par chromatographie en phase gazeuse après transméthylation.

\section{Analyse statistique}

Les compositions en acides gras de 30 sujets ayant participé aux 3 régimes ont été comparées en utilisant le test $\mathrm{t}$ (apparié) de Student.

\section{Résultats et discussion}

Effets comparés des régimes sur la composition en acides gras des esters de cholestérol plasmatiques

Le tableau rassemble les valeurs (moyennes et écarts types) des acides gras des EC du plasma des sujets, au terme de 4 mois des 3 régimes testés. Les différences de résultats observées entre deux régimes sont appréciées par les valeurs de $p$ mentionnées dans ce tableau. L'examen de la composition globale en acides gras saturés (AGS), acides gras mono-insaturés (AGMI) et acides gras poly-insaturés $n-6$ et $n-3$ (AGPI) montre une similitude parfaite entre les régimes OL-11 \% et OL- $16 \%$ ; le régime $\mathrm{OL}-13 \%$ induit, quant à lui, une différence significative au niveau des AGMI et AGPI, le pourcentage d'AGS (environ $13 \%$ des acides gras) étant identique avec les 3 régimes. Avec OL-13 $\%$,on note une baisse des proportions d'AGMI (20,8 \% contre $24 \%)$ compensée par une hausse des AGPI (66\% contre $63 \%)$. Ces différences sont dues essentiellement à l'acide oléique pour les AGMI et à l'acide linoléique pour les AGPI. Par rapport aux régimes OL-11 \% et OL-16\%, le régime OL- $13 \%$ contribue à l'augmentation des proportions de 18:2n-6 (52-53\% vs $55 \%$ ) et à la baisse de celles du 18:1n-9 (19,4 \% vs 16,4 \%). Le fait que les EC accumulent moins d'oléate de cholestérol avec un régime qui pourtant en apporte plus (OL-13\% contre OL-11 \%) pourrait s'expliquer par les proportions 18:1n-9/18:2n-6 de chaque régime. En effet, le régime OL-13\% apporte plus de 18:2n-6 que le régime OL-11 \% (4,5\% AET vs 3,6 \%) ; ainsi le rapport 18:1n-9/18:2n-6 est respectivement égal 
à 2,8 (OL-13 \%) et 3,1 (OL-11 \%). Les travaux de Rudel et al. [4-6] ont montré l'influence des apports respectifs en 18:1n-9 et 18:2n-6 sur la composition des EC du plasma.

Le niveau d'incorporation de l'acide alphalinolénique dans les EC se situe entre 0,63 (OL-16 \%) et 0,7 $\%$ (OL-11 \%) par rapport aux acides gras totaux. Les différences qui portent sur les pourcentages d'EPA accumulé avec ces 3 régimes pourraient s'expliquer soit par des apports respectifs en acides gras n-3 à très longues chaînes différents, soit par la synthèse endogène d'EPA à partir du 18:3n-3.

Influence du rapport linoléique/ alphalinolénique du régime sur le niveau d'incorporation de l'acide alphalinolénique dans les esters de cholestérol plasmatiques

La troisième édition des ANC préconise un rapport $R$ de 5 entre les apports de 18:2n-6 et 18:3n-3. Actuellement, I'alimentation moyenne des Français est caractérisée par un $R$ de 14 , dû principalement à un faible apport en 18:3n-3 (0,3\% AET contre 0,8\% recommandé) [24, 25]. L'étude d'intervention que nous avons conduite permet d'atteindre ou presque ce rapport (soit $R=5-8$ ). L'objectif de l'analyse suivante (figure) est d'examiner, en fonction de conditions nutritionnelles différentes par leurs apports respectifs en AGMI et/ou AGPIn-6 et/ou n-3, l'évolution des niveaux d'incorporation du 18:3n-3 dans les EC du plasma, par rapport à la zone souhaitable eu égard aux risques MCV (cf. paragraphe introductif). À cette fin, nous avons comparé les situations d'une population française [24], dont l'alimentation est caractérisée par un rapport 18:2n-6/18:3n-3 égal à 14 , et d'une population similaire, avec $R=19,5$ [18], à celles des sujets de l'étude (régimes avec $R=6$ et 8). Sur la figure, sont reportés les pourcentages en 18:3n-3 et 20:5n-3 présents dans les EC de ces populations. L'examen de cette figure appelle les remarques suivantes : (i) le niveau d'incorporation de $18: 3 n-3$ dans les EC est identique $(=0,4 \%$ des acides gras) pour des rapports $18: 2 n-6 / 18: 3 n-3$ élevés ( $R=19,5$ et 14$)$. On remarque qu'il augmente pour atteindre $0,7 \%$ lorsque ce rapport diminue (de 14 à 6,4). Entre les conditions caractérisées par un $R$ élevé et le régime OL-11 \% $(R=6,4)$, le gain en 18:3n-3 des EC est de $57 \%$ (ii) ; parallèlement à l'augmentation des proportions de 18:3n-3, on peut noter que celles d'EPA sont doublées ( $1,2 \%$ vs $0,6 \%$ ). L'apport en acides gras poly-insaturés n-3 à très longues chaînes entre les 2 régimes testés (OL-11 \% et OL-16 \%) et les populations contrôles étant identique $(0,04 \% A E T)$, cet incrément d'EPA serait dû à sa biosynthèse à partir du 18:3n-3, dont la biodisponibilité serait améliorée par un rapport 18:2n-6/18:3n-3 plus faible (6-8 vs 14-20) d'une part, et par la forme d'apport de l'acide alphalinolénique dans ces régimes (i.e. huile de colza dont le 18:3n-3 est préférentiellement situé en position sn 2 des TG), d'autre part.

\section{CONCLUSION}

En conclusion, les régimes proposés et testés ont permis d'accroître le niveau de 18:3n-3 dans les EC du plasma d'en moyenne $57 \%$ (de 0,4 à 0,6-0,7 \% des acides gras) et de doubler celui d'EPA. En outre, on vérifie que lorsque les proportions d'acide oléique par rapport à celles de l'acide linoléique diminuent dans le régime, l'incorporation de ce dernier dans les EC est meilleure. Parallèlement à ces effets sur la composition en acides gras poly-insaturés des esters de cholestérol, ces régimes se sont avérés conformes aux attentes en terme de profil des paramètres prédictifs du risque cardiovasculaire (premier exposé de ce dossier). 


\section{Remerciements}

Les auteurs remercient Laurence Fonseca et Nadège Dubourdieu pour leur participation technique efficace.

Ce travail a bénéficié du soutien financier de I'Organisation Nationale Interprofessionnelle des Oléagineux (ONIDOL) et du Centre Technique Interprofessionnel des Oléagineux Métropolitains (CETIOM).

\section{REFERENCES}

1. MARTIN A (2001). Apports nutritionnels conseillés pour la population française, Tec \& Doc, ed. France : CNERNA-CNRS, AFSSA.

2. GRUNDY SM (1997). What is the desirable ratio of saturated, polyunsaturated, and monounsaturated fatty acids in the diet? Am J Clin Nutr, 66 (Suppl.) : 988S-90.

3. JEWETT B (2002). MUFA versus PUFA: scientists disagree on nutritional importance of these fatty acids. Inform, $13:$ 376-9.

4. RUDEL LL, PARKS JS, SAWYER JK (1995). Compared with dietary monounsaturated and saturated fat, polyunsaturated fat protects african green monkeys from coronary artery atherosclerosis.

Arterioscler Throm Vasc Biol, 15 : 2101-10.

5. RUDEL LL, HAINES J, SAWYER JK, SHAH R, WILSON MS, et al. (1997). Hepatic origin of cholesteryl oleate in coronary artery atherosclerosis in african green monkeys. Enrichment by dietary monounsaturated fat. J Clin Invest, 100 : 74-83.

6. RUDEL LL, KELLEY K, SAWYER JK, SHAH R, WILSON MS (1998). Dietary monounsaturated fatty acids promote aortic atherosclerosis in LDL. Receptor-Null, human ApoB100-overexpressing transgenic mice. Arterioscler Throm Vasc Biol, 18 : 1818-27.

7. DOLECEK TA, GRANDITS G (1991). Dietary polyunsaturated fatty acids and mortality in the multiple risk factor intervention trial (MRFIT). In : SIMOPOULOS AP, KIFER RR, MARTIN RE, BARLOW SM, eds. Health effects of omega 3 fatty acids in sea foods. World Rev Nutr Basel Karger, 66 : 205-16.

8. ASCHERIO A, RIMM EB, GIOVANNUCCI FL, SPEIGELMAN D, STAMPFER M, et al. (1996) Dietary fat and risk of coronary heart disease in men: cohort follow-up study in the United States. Br Med J, 313 : 84-90.

9. HU FB, STAMPFER M, MANSON JE, RIMM EB, WOLK A, et al (1999). Dietary intake of a linolenic acid and risk of fatal ischemic heart disease among women. Am J Clin Nutr, 69 : 890-97.

10. SIMON JA, FONG J, BERNERT JT, BROWNER WS (1995). Serum fatty acids and risk of stroke. Stroke, $26: 778-82$. 
11. RENAUD SC (2001). Diet and stroke. J Nutr Health Aging, 5 : 167-72.

12. SANDKER GW, KROMHOUT D, ARAVANIS C, BLOEMBERG BP, MENSINK RF, et al. (1993). Serum cholesteryl ester fatty acids and their relation with serum lipids in elderly men in Crete and The Netherlands. Eur J Clin Nutr, 47 : 201-8.

13. BEMELMANS WJ, MUSKIET FA, FESKENS EJ, de VRIES JH, BROER J, et al. (2000). Associations of alpha-linolenic acid and linoleic acid with risk factors for coronary heart disease. Eur J Clin Nutr, 54 : 865-71.

14. LEYTON J, DRURY P, CRAWFORD RA (1987) Differential oxidation of saturated and unsaturated fatty acids in vivo in the rat. Brit J Nutr, $57: 383-93$.

15. VERMUNT SH, MENSINK RP, SIMONIS MM, HORNSTRA G (2000). Effects of dietary alpha-linolenic acid on the conversion and oxidation of 13C-alpha-linolenic acid. Lipids, 35 : 137-42.

16. BRENNA JT (2002). Efficiency of conversion of alpha-linolenic acid to long chain n-3 fatty acids in man. Curr Opin Clin Nutr Metab Care, 5 : 127-32.

17. CLOUET P, NIOT I, BEZARD J (1989). Pathway of alpha-linolenic acid through the mitochondrial outer membrane in the rat liver and influence on the rate of oxidation. Comparison with linoleic and oleic acids. Biochem J, $263:$ 867-73.

18. MANTZIORIS E, JAMES MJ, GIBSON RA, CLELAND LG (1994). Dietary substitution with an alphalinolenic acid-rich vegetable oil increases eicosapentaenoic acid concentrations in tissues. Am J Clin Nutr, 59 : 1304-9.

19. VALSTA LM, SALMINEN I, ARO A, MUTANEN M (1996). Alpha-linolenic acid in rapeseed oil partly compensates for the effect of fish restriction on plasma long chain n-3 fatty acids. Eur J Clin Nutr, 50 : 229-35.

20. BEMELMANS WJE, BROER J, FESKENS EJM, SMIT AJ, MUSKIET FAJ, et al. (2002). Effect of an increased intake of alpha-linolenic acid and group nutritional education on cardiovascular risk factors: the Mediterranean Alpha-linolenic Enriched Groningen Dietary Intervention (MARGARIN) study. Am J Clin Nutr, 75 : 221-7.

21. MA J, FOLSOM AR, SHAHAR E, ECKFELDT JH (1995). Plasma fatty acid composition as an indicator of habitual dietary fat intake in middle-aged adults. Am J Clin Nutr, 62 : 564-71.

22. BABIN F, RODRIGUEZ A, SARDA P, VANDEPUTTE B, MENDY F, et al (2000). Alpha-linolenic acid in cholesterol esters: a marker of alpha-linolenic acid intake in newborns. Eur J Clin Nutr, 54 : 840-3.

23. COMBE N, JUDDE A, BILLEAUD C, BOUE C, TURON F, ENTRESSANGLES B, DALLAY D, LENG JJ (1998). Distribution of dietary trans isomers of essentiel fatty acids in blood lipid classes. In : RIEMERSMA RA, AMSTRONG RA, KELLY RW, WILSON R, eds. Proceedings of the Fourth International Congress on Essential Fatty Acids and Eicosanoids. AOCS Press, 239-42.

24. COMBE N, BOUÉ C (2001). Apports alimentaires en acides linoléique et alphalinolénique d'une population d'Aquitaine. $O C L, 8: 118-21$. 
25. HULSHOF KFAM, VAN ERP-BAART MA, ANTTOLAINEN M, et al. (1999). Intake of fatty acids in western Europe with emphasis on trans fatty acids: the TRANSFAIR study. Eur J Clin Nutr, 53 : 143-57.

Illustrations

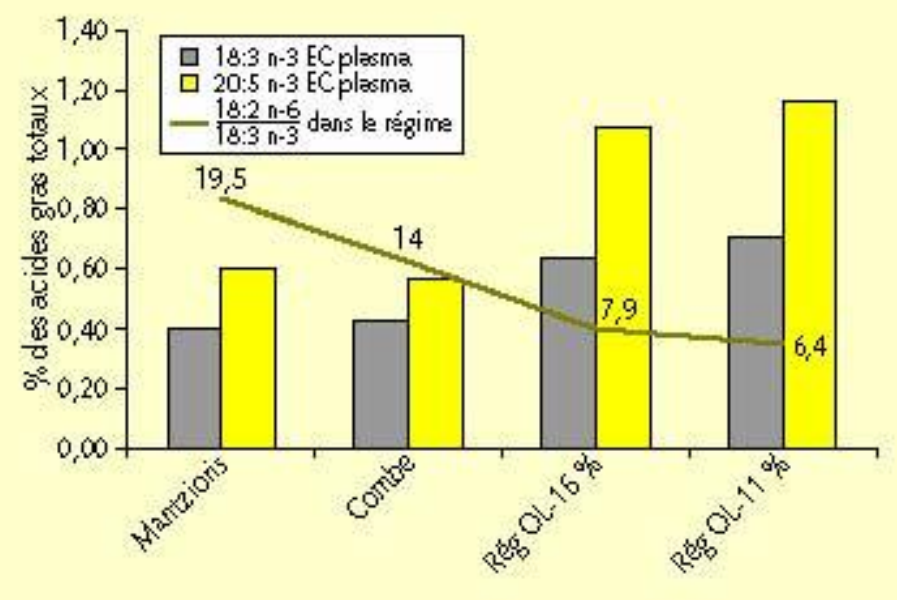

Figure. Influence du rapport 18:2 n-6/18:3 n-3 du régime sur le niveau d'incorporation des acides 18:3n-3 et 20:5 n-3 dan les esters de cholestérol plasmatiques.

Tableau. Composition en acides $g$ as (\% pds des acides $g$ as totoux) des esters de cholestérol du plesma.

\begin{tabular}{|c|c|c|c|c|c|c|c|c|c|}
\hline \multirow[b]{2}{*}{ Acid es gras } & \multicolumn{2}{|c|}{$\begin{array}{l}\text { Régime (OL-11 \%) } \\
\qquad(\mathrm{n}=30)\end{array}$} & \multicolumn{2}{|c|}{$\begin{array}{l}\text { Régime (OL-13\%) } \\
\qquad(n=30)\end{array}$} & \multicolumn{2}{|c|}{$\begin{array}{l}\text { Régime (OL-16\%) } \\
\qquad(n=30)\end{array}$} & \multicolumn{3}{|c|}{ P (test apparié) } \\
\hline & Moyenne & Ecart type & Moyenne & Ecart-type & Moyenne & Ecart type & $11-13 \%$ & $11-16 \%$ & $13-16 \%$ \\
\hline AGS totaux & 13,25 & 1,09 & 13,10 & 3,61 & 12,7 & 1,01 & 0,853 & 0,021 & 0,651 \\
\hline AGMI totaux & 24,12 & 2,26 & 20,79 & 3,40 & 23,74 & 1,82 & 0,000 & 0,427 & 0,000 \\
\hline AGPI totaux & 62,40 & 3,04 & 66,00 & 6,87 & 63,31 & 2,29 & 0,010 & 0,157 & 0,034 \\
\hline $18: 1 n-9$ & 19,42 & 1,85 & 16,39 & 2,86 & 19,47 & 1,81 & 0,000 & 0,909 & 0,000 \\
\hline $18: 2 n-6$ & 51,67 & 4,04 & 55,12 & 6,60 & 52,96 & 2,81 & 0,013 & 0,038 & 0,043 \\
\hline $20: 4 n-6$ & 6,61 & 1,50 & 6,27 & 1,68 & 6,45 & 1,42 & 0,394 & 0,305 & 0,108 \\
\hline $18: 3 n-3$ & 0,70 & 0,11 & 0,68 & 0,12 & 0,63 & 0,12 & 0,514 & 0,017 & 0,030 \\
\hline $20: 5 n-3$ & 1,15 & 0,31 & 1,73 & 0,76 & 1,07 & 0,35 & 0,000 & 0,094 & 0,000 \\
\hline $22: 6 n-3$ & 0,84 & 0,20 & 0,91 & 0,21 & 0,80 & 0,14 & 0,226 & 0,127 & 0,002 \\
\hline
\end{tabular}

AGS : acides qras saturés ; AGMl : acides qras irsaturés ; AGPI : acides qras polyirøaturés $n-6$ et $n-3$. 